This item was submitted to Loughborough's Research Repository by the author.

Items in Figshare are protected by copyright, with all rights reserved, unless otherwise indicated.

\title{
Honeycomb and triangular domain wall networks in heteroepitaxial systems
}

PLEASE CITE THE PUBLISHED VERSION

https://doi.org/10.1063/1.4948370

PUBLISHER

(c) the Authors. Published by AIP Publishing

VERSION

VoR (Version of Record)

LICENCE

CC BY-NC-ND 4.0

REPOSITORY RECORD

Elder, Ken R., Z. Chen, K.L.M. Elder, Petri Hirvonen, S.K. Mkhonta, S.C. Ying, E. Granato, Zhi-Feng Huang, and Tapio Ala-Nissila. 2019. "Honeycomb and Triangular Domain Wall Networks in Heteroepitaxial Systems". figshare. https://hdl.handle.net/2134/28202. 


\section{Honeycomb and triangular domain wall networks in heteroepitaxial systems}

K. R. Elder, Z. Chen, K. L. M. Elder, P. Hirvonen, S. K. Mkhonta, S.-C. Ying, E. Granato, Zhi-Feng Huang, and T. Ala-Nissila

Citation: The Journal of Chemical Physics 144, 174703 (2016);

View online: https://doi.org/10.1063/1.4948370

View Table of Contents: http://aip.scitation.org/toc/jcp/144/17

Published by the American Institute of Physics

\section{Articles you may be interested in}

Free Energy of a Nonuniform System. I. Interfacial Free Energy

The Journal of Chemical Physics 28, 258 (2004); 10.1063/1.1744102

Crystal nucleation as the ordering of multiple order parameters

The Journal of Chemical Physics 145, 211801 (2016); 10.1063/1.4962166

A Navier-Stokes phase-field crystal model for colloidal suspensions

The Journal of Chemical Physics 142, 154904 (2015); 10.1063/1.4918559

Role of modulus mismatch in vertically aligned nanocomposite formation during spinodal decomposition in constrained films

Applied Physics Letters 103, 141903 (2013); 10.1063/1.4823700

Epitaxial growth and structural property of graphene on $\mathrm{Pt}(111)$

Applied Physics Letters 98, 033101 (2011); 10.1063/1.3543624

Phase field microelasticity theory and modeling of elastically and structurally inhomogeneous solid Journal of Applied Physics 92, 1351 (2002); 10.1063/1.1492859

\section{AIP | the Joumal of Chemical Physics}




\title{
Honeycomb and triangular domain wall networks in heteroepitaxial systems
}

\author{
K. R. Elder, ${ }^{1}$ Z. Chen, ${ }^{1,2}$ K. L. M. Elder, ${ }^{3}$ P. Hirvonen, ${ }^{3}$ S. K. Mkhonta, ${ }^{4,5}$ S.-C. Ying, ${ }^{6}$ \\ E. Granato, ${ }^{6,7}$ Zhi-Feng Huang, ${ }^{5}$ and T. Ala-Nissila ${ }^{3,6}$ \\ ${ }^{1}$ Department of Physics, Oakland University, Rochester, Michigan 48309, USA \\ ${ }^{2}$ Department of Applied Physics, Northwestern Polytechnical University, Xi'an 710129, \\ People's Republic of China \\ ${ }^{3}$ Department of Applied Physics and COMP Centre of Excellence, Aalto University School of Science, \\ P.O. Box 11000, FI-00076 Aalto, Finland \\ ${ }^{4}$ Department of Physics, University of Swaziland, Private Bag 4, Kwaluseni, Swaziland \\ ${ }^{5}$ Department of Physics and Astronomy, Wayne State University, Detroit, Michigan 48201, USA \\ ${ }^{6}$ Department of Physics, Brown University, P.O. Box 1843, Providence, Rhode Island 02912, USA \\ ${ }^{7}$ Laboratório Associado de Sensores e Materiais, Instituto Nacional de Pesquisas Espaciais, \\ 12227-010 São José dos Campos, SP, Brazil
}

(Received 2 February 2016; accepted 19 April 2016; published online 2 May 2016)

\begin{abstract}
A comprehensive study is presented for the influence of misfit strain, adhesion strength, and lattice symmetry on the complex Moiré patterns that form in ultrathin films of honeycomb symmetry adsorbed on compact triangular or honeycomb substrates. The method used is based on a complex Ginzburg-Landau model of the film that incorporates elastic strain energy and dislocations. The results indicate that different symmetries of the heteroepitaxial systems lead to distinct types of domain wall networks and phase transitions among various surface Moiré patterns and superstructures. More specifically, the results show a dramatic difference between the phase diagrams that emerge when a honeycomb film is adsorbed on substrates of honeycomb versus triangular symmetry. It is also shown that in the small deformation limit, the complex Ginzburg-Landau model reduces to a two-dimensional sine-Gordon free energy form. This free energy can be solved exactly for one dimensional patterns and reveals the role of domains walls and their crossings in determining the nature of the phase diagrams. Published by AIP Publishing. [http://dx.doi.org/10.1063/1.4948370]
\end{abstract}

\section{INTRODUCTION}

Understanding and predicting the complex superstructures that emerge during surface ordering and the associated phase transitions between these structures have been of both fundamental and practical importances for decades. Such systems, which are comprised of ultrathin strained films deposited on compact ordered surfaces, usually exhibit novel and tunable electronic, optical, catalytic, and magnetic properties. Traditional systems include the case of rare-gas (e.g., $\mathrm{Kr}$, $\mathrm{Ar}$, or $\mathrm{Xe}$ ) absorbed monolayers on graphite $\mathrm{e}^{1-5}$ and metal-metal heteroepitaxial overlayer systems (e.g., $\mathrm{Cu}$, $\mathrm{Ni}$, or $\mathrm{Co}$ on $\mathrm{Ru}(0001)$ or $\mathrm{Pd}(111)$ and $\mathrm{Pb}$ on $\left.\mathrm{Si}(111)^{6-14}\right)$. Most recently, the emergence of novel two-dimensional materials such as graphene, hexagonal Boron Nitride (h-BN), and transition metal dichalcogenides (TMDs) ${ }^{15-20}$ and their epitaxial growth on solid substrates has re-ignited interest in this field. While these systems involve very different atomistic details, the fundamental mechanisms underlying their surface ordering processes are largely governed by the interplay of three pivotal factors: misfit strain $(\varepsilon)$, adhesion strength $\left(V_{0}\right)$, and the relative lattice symmetry of the overlayer film with respect to the substrate surface. This latter mechanism plays a major role in influencing both the symmetry of the incommensurate superstructures or Moiré patterns that form in the films and the nature of the phase transitions between various incommensurate patterns and commensurate states.
The focus of this work is on the ordering of honeycomb (H) films on compact triangular $(\mathrm{T})$ and honeycomb $(\mathrm{H})$ substrates, where the magnitude of the lattice constants of the substrate and film is reasonably close so as to avoid larger unit commensurate phases. To clarify the terminology used in this paper the terms $\mathrm{H}$ or $\mathrm{T}$ substrates refer to substrates where the surface atoms form a honeycomb or triangular lattice, respectively. Similarly a $\mathrm{H}$ or $\mathrm{T}$ film refers to a monolayer that has density maxima that form a honeycomb or triangular lattice in commensurate domains as well as in the completely incommensurate state.

In this work it is assumed that the substrate surface atoms represent adsorption potential maxima and the hollow sites potential minima. For triangular substrates both the fcc and hcp hollow sites represent potential minima. This means neglecting the small difference between these two types of adsorption sites due to the influence of the sub-surface layers of the substrate. As can be seen in Fig. 1, under these conditions the adsorption sites for the triangular substrate form a honeycomb lattice whereas the adsorption sites for a honeycomb substrate form a triangular (hexagonal) lattice. Physically the H-T system is relevant to the 2D ordering of graphene, h-BN, or TMD films on compact metallic (111) or (0001) substrate surfaces, while the H-H system is relevant to the graphene ordering on h-BN substrate.

Developing a comprehensive understanding of the complex superstructures that form in these systems is 


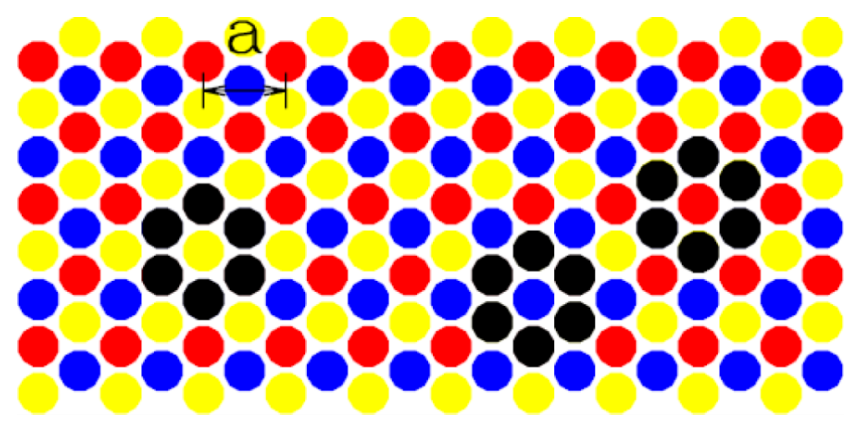

FIG. 1. Schematics of the surface ordering. For a compact triangular (111) or (0001) substrate the yellow dots correspond to the substrate potential maxima and the red and blue to hcp and fcc surface absorption (potential minima) locations, respectively. In this instance the honeycomb ring of six black atoms on the left is in the lowest-energy commensurate state and the two rings of black atoms on right are in higher energy commensurate states. For a substrate of honeycomb symmetry the red and blue locations correspond to potential maxima and the yellow to potential minima. In this case the ring on the left is a high energy incommensurate state and the two lower energy states on the right are equivalent.

hampered by the multiple scales involved, from microscopic dislocations in the crystalline lattice to nano or even micron scales of surface patterning. Continuum descriptions have been used to examine the nature of the commensurateincommensurate (C-I) transitions from striped or hexagonal phase to a commensurate phase, showing a continuous transition in the former case and a discontinuous transition for the latter. ${ }^{1-5}$ However, these descriptions neglect atomistic details of the crystalline lattice which are expected to play an important role at large strains and are not well understood in the study of C-I transitions. Unfortunately it is difficult to employ atomistic simulation methods as the length scale of the patterns diverge at the C-I transition and is inversely proportional to $\varepsilon$ at small $V_{0}$. Here a study is presented of the ordering of 2D strained overlayers of honeycomb symmetry on either triangular or honeycomb substrate, emphasizing both atomistic and long range features of the system. The problem of multiple length scales is resolved by employing a complex amplitude model ${ }^{21-26}$ based on the phase-field crystal (PFC) method. ${ }^{27-30}$ This approach has been applied to the modeling of a large variety of elastic and plastic systems from nano to micron length scales. ${ }^{11,12,29-39}$ The results of this work elucidate the influence of overlayer lattice symmetry on the nature of phase transitions between various superstructured phases. Interestingly the results indicate a dramatic difference between the $\mathrm{H}-\mathrm{H}$ and $\mathrm{H}-\mathrm{T}$ systems. This can be understood in terms of different energy states of a honeycomb ring commensurate with a triangular or honeycomb substrate as illustrated in Fig. 1. For a compact triangular (111) or (0001) substrate, a ring of six black atoms is in a lowest-energy commensurate state when they are all adsorbed on the fcc or hcp hollow sites. There are two higher-energy commensurate states when the adatoms are half on atop sites and half in fcc or hcp hollow sites. For a substrate of honeycomb symmetry, adsorption of the ring on atop sites is a high energy state whereas there are two lower energy states with half of the ring on top sites and half in hollow sites. This difference in energy of states of a commensurate ring in turn leads to different energies at junctions or domain wall crossings for the two types of adsorption systems.

This paper is organized in the following fashion. In Sec. II the methodology used for the study is outlined, followed in Sec. III by a presentation of the results of a numerical investigation of the patterns that form as a function of misfit strain and adhesion strength. In Sec. IV analytic calculations in the small deformation limit are presented to elucidate the nature of the transitions observed numerically. Finally a discussion of the results and some conclusions are given in Sec. V.

\section{METHOD}

To model the ordering process a method introduced in prior publications ${ }^{11,12}$ for a triangular film ordering on a triangular substrate will be exploited. The model is based on the complex amplitude equations derived originally from the PFC model by Goldenfeld and collaborators as described in Refs. 21-26. In this approach the dimensionless atomic number density field, $n$, is represented in terms of a set of complex amplitudes, $\eta_{\mathrm{klm}}$. Equations of motion for the three lowest order amplitudes needed to construct a system of triangular symmetry were derived. For simplicity the substrate is approximated by a rigid potential which should be appropriate for ultrathin films and particularly for graphene ordering on metallic surface where the interaction is generally weak. Extension of the amplitude model to include a fixed surface potential, $V(\vec{r})$, was done in prior work. ${ }^{11,12}$ For a compact (111) metallic surface this potential can be written as

$$
V=V_{0} \sum_{k l m} e^{i \vec{G}_{k l m}^{s} \cdot \vec{r}}+\text { c.c. },
$$

where $\quad \vec{G}_{k l m}^{s}=k \vec{q}_{1}+l \vec{q}_{2}+m \vec{q}_{3}, \quad\left(\vec{q}_{1}, \vec{q}_{2}, \vec{q}_{3}\right) \quad$ are principle reciprocal lattice vectors of the substrate potential, and $\mathrm{klm}$ are the Miller indices. For a triangular Bravais lattice, $\vec{q}_{1}=q(-\sqrt{3} / 2,-1 / 2,0), \vec{q}_{2}=q(0,1,0)$, and $\vec{q}_{3}=(0,0,0)$, and $q$ sets the length scale of the system. To construct a triangular lattice with minimal number of plane waves, three wave vectors are needed, corresponding to $(\mathrm{klm})=(100),(010)$,

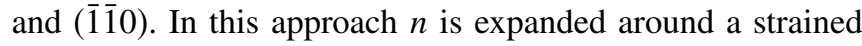
state, i.e.,

$$
n=\sum_{k l m}\left[\eta_{k l m} e^{i \vec{G}_{k l m}^{s} \cdot \vec{r}}+\text { c.c. }\right],
$$

where the equilibrium reciprocal lattice vectors of the film are such that $\vec{G}_{k l m}^{f}=\vec{G}_{k l m}^{s} / \alpha$ for a misfit strain of $\varepsilon=1-\alpha$. In the PFC model the dynamics of $n$ is governed by

$$
\frac{\partial n}{\partial t}=\Gamma \nabla^{2} \frac{\delta F}{\delta n},
$$

where $\Gamma$ sets the time scale and the free energy functional is given by

$$
\begin{aligned}
F=\frac{\mathcal{F}}{k_{B} T \bar{\rho}}= & \int d \vec{r}\left[\Delta B \frac{n^{2}}{2}+B^{x} \frac{n}{2}\left(1+\nabla^{2}\right)^{2} n\right. \\
& \left.-t \frac{n^{3}}{3}+v \frac{n^{4}}{4}+V n\right] .
\end{aligned}
$$

Here $k_{B}$ is Boltzmann constant, $T$ is temperature, and $\bar{\rho}$ is the average atomic number density. The parameters $\Delta B$, 
$B^{x}, t$, and $v$ are phenomenological as described in Refs. 25, 26 , and 29. In brief, $B^{x}$ is related to the elastic moduli, $\Delta B$ is related to a temperature difference from melting, and $t$ and $v$ control the amplitude of density fluctuations. As shown in prior work, ${ }^{21-26}$ if the lowest-order wave vectors $((k l m)=(100),(010)$, and $(\overline{1} \overline{1} 0))$ are used to describe a film of triangular symmetry the equation of motion for $\eta_{k l m}$ can be written as

$$
\frac{\partial \eta_{k l m}}{\partial t}=-\Gamma \frac{\delta F_{\eta}}{\delta \eta_{k l m}^{*}},
$$

where

$$
\begin{aligned}
F_{\eta}= & \int d \vec{r}\left[\sum_{k l m}\left(B^{x}\left|\mathcal{G}_{k l m} \eta_{k l m}\right|^{2}-\frac{3 v}{2}\left|\eta_{k l m}\right|^{4}\right)\right. \\
& +\frac{\Delta B}{2} A^{2}+\frac{3 v}{4} A^{4}-2 t\left(\prod_{k l m} \eta_{k l m}+\text { c.c. }\right) \\
& \left.+V_{0}\left(\sum_{k l m} \eta_{k l m}+\text { c.c. }\right)\right] .
\end{aligned}
$$

Here $A^{2} \equiv 2 \sum_{k l m}\left|\eta_{k l m}\right|^{2}$ and $\mathcal{G}_{k l m} \equiv \nabla^{2}+2 i \alpha \vec{G}_{k l m}^{f} \cdot \vec{\nabla}+1$ $-\alpha^{2}$.

It is useful to note that the equilibrium states of this free energy functional are described by $\eta_{k l m}^{e q}=\phi$ where

$$
\phi=\frac{t \pm \sqrt{t^{2}-15 v \Delta B_{0}}}{15 v} .
$$

The "+" solution is the minimum energy solution when $t>0$, and reconstruction of the density field from Eq. (2) gives rise to a pattern in which the density maxima form a triangular pattern. The "-" solution is the equilibrium solution when $t<0$ and the reconstructed density is a honeycomb array of maxima. Thus for the purposes of this work $t>0$ will be used to describe a triangular film and $t<0$ for a honeycomb film. Another way to see this relation between the honeycomb and triangular film through a change of sign of the density expansion is to start with the density maxima centered on a honeycomb lattice described by a two-dimensional triangular Bravais lattice with a two-point basis, i.e.,

$$
n=\sum_{j=1}^{2} \sum_{k l m} \eta_{k l m} e^{i \vec{G}_{k l m}^{s} \cdot\left(\vec{r}-\vec{r}_{j}\right)}+\text { c.c. }
$$

where $\overrightarrow{\mathrm{r}}_{1}=0, \overrightarrow{\mathrm{r}}_{2}=\vec{\delta}=(0,-4 \pi / 3 q, 0)$, and the $\vec{G}_{k l m}^{s}$ are for a triangular lattice as described previously. If only the basic wave vectors $(k l m)=(100),(010)$, and $(\overline{1} \overline{1} 0)$ are used to described the triangular lattice then it is straightforward to show that the equilibrium states are given by

$$
\begin{aligned}
n & =2 \phi \sum_{k l m}\left[\cos \left(\vec{G}_{k l m}^{s} \cdot \vec{r}\right)+\cos \left(\vec{G}_{k l m}^{s} \cdot\left(\vec{r}-\vec{r}_{2}\right)\right)\right] \\
& =-2 \phi \sum_{k l m}\left[\cos \left(\vec{G}_{k l m}^{s} \cdot\left(\vec{r}+\vec{r}_{2}\right)\right)\right] .
\end{aligned}
$$

which corresponds to the solution of Eq. (7) with a negative amplitude. This same argument applies to the substrate potential. If $V_{0}>0 \quad(<0)$ it describes a triangular array of potential maxima (minima) and a honeycomb array of potential minima (maxima). These results taken together with the free energy given in Eq. (6) imply that the solutions for which both $t$ and $V_{0}$ are positive (i.e., a triangular film on a triangular surface, the T-T system) are identical to the solutions when they are both negative (i.e., a honeycomb film on a honeycomb substrate, the $\mathrm{H}-\mathrm{H}$ system) except for a change in the sign of the amplitudes. For this reason the prior results $^{11,12}$ for a T-T system can be used to describe the $\mathrm{H}-\mathrm{H}$ case and to compare with the honeycomb-on-triangular $(\mathrm{H}-\mathrm{T})$ scenario presented in Sec. III.

\section{NUMERICAL RESULTS}

Simulations were conducted to determine the equilibrium phase diagram of the H-T system by numerically evolving
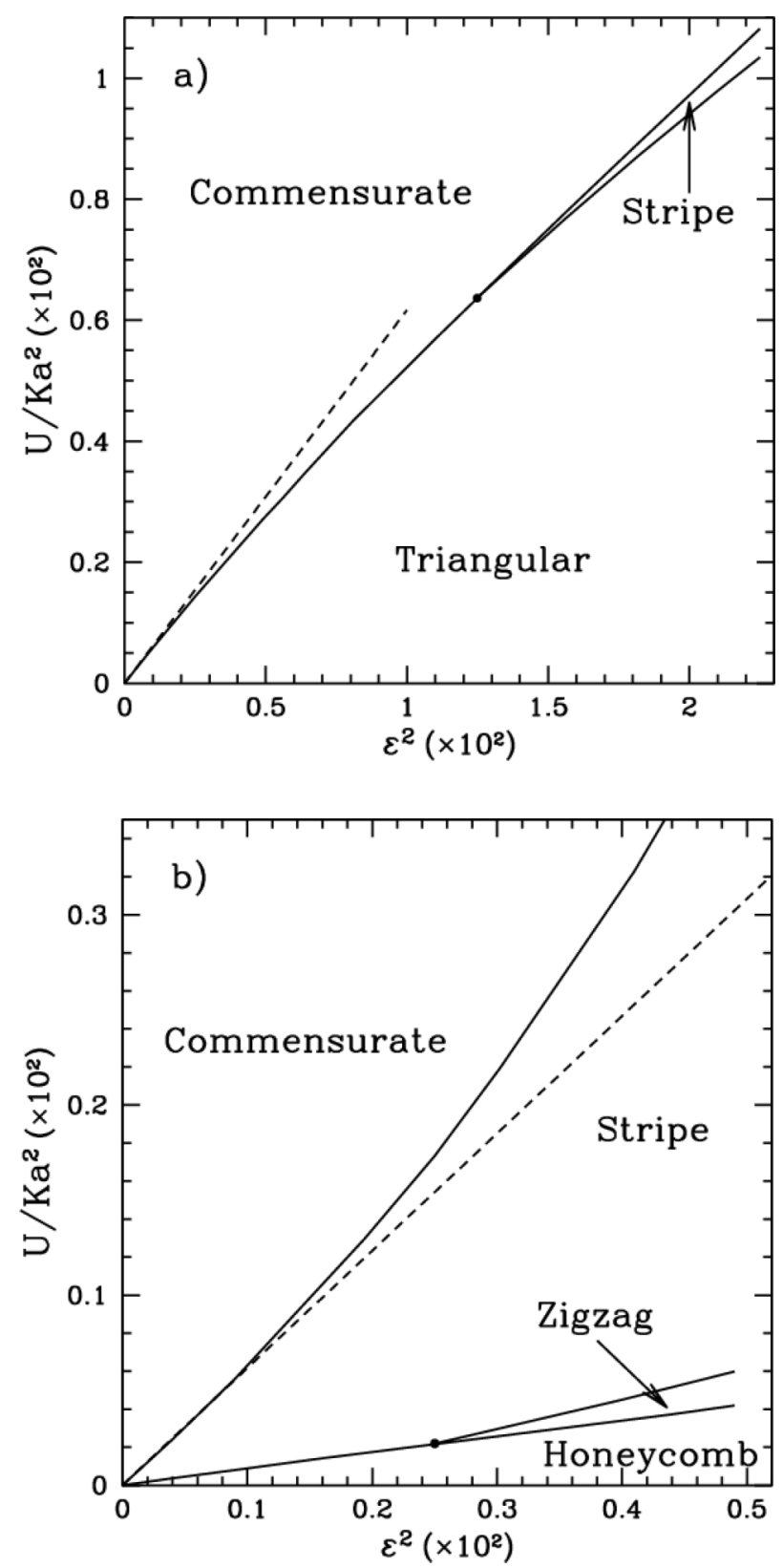

FIG. 2. Phase diagrams for (a) H-T and (b) H-H systems. The dashed lines correspond to $U / K a^{2}=\pi^{2} \varepsilon^{2} / 16$. (b) is redrawn from Ref. 12 for the $\mathrm{H}-\mathrm{H}$ system. The black dots correspond to triple points. 

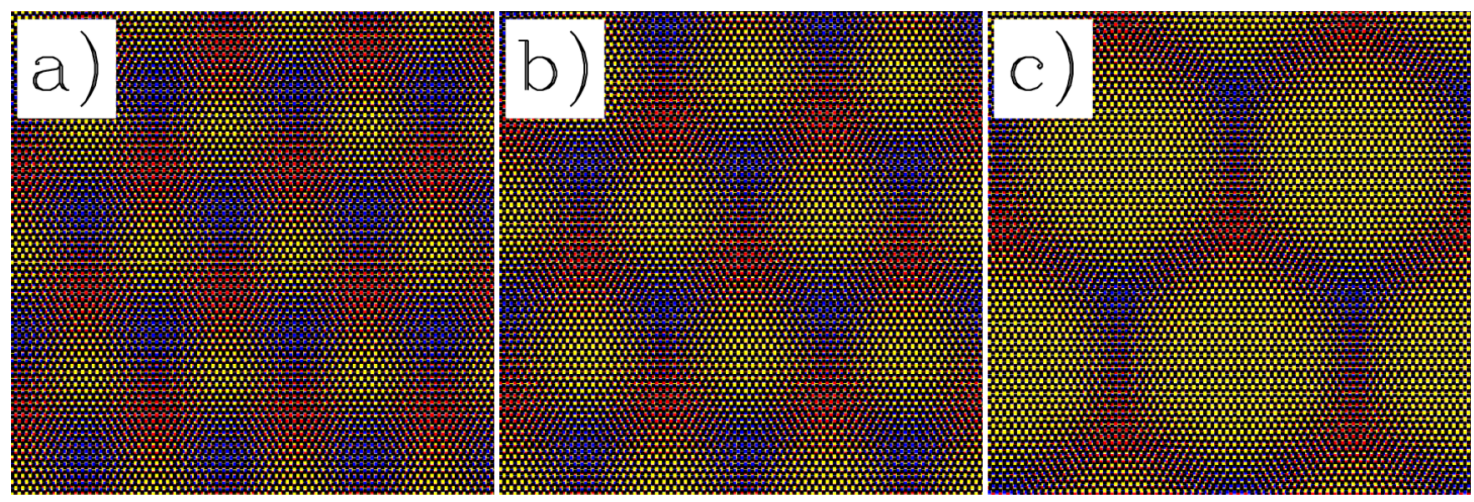

FIG. 3. Examples of equilibrium triangular Moiré patterns for the H-T system at $\varepsilon=5 \%$ and $U / K a^{2}=1.4375 \times 10^{-4}, 7.1876 \times 10^{-4}$, and $1.4088 \times 10^{-3}$ in (a)-(c), respectively. Light (yellow) and dark (red and blue) areas correspond to commensurate and incommensurate regions, respectively.

Eq. (5) until the free energy was no longer changing in time. The initial conditions were a uniformly strained film for the two dimensional patterns and a uniform strain in one direction for the stripe states. In all cases periodic boundary conditions were employed. The parameters used for the simulations were $\left(\Delta B, B^{x}, t, v\right)=(0.02,0.98, \mp 1 / 2,1 / 3)$. A semi-implicit spectral relaxation method was used with a grid spacing of $\Delta x=2.0$ and time step of $\Delta t=0.5$. The system size was determined by the size of the pattern being considered and varied to determine which length minimized the free energy per unit area. For convenience the selected states were determined as a function of a dimensionless adhesion strength, $U /\left(K a^{2}\right) \sim V_{0}$, and misfit strain $\varepsilon$. The expressions of adhesion energy $U$, elastic modulus $K$, and lattice constant $a$ and the relationship between $U /\left(K a^{2}\right)$ and $V_{0}$ will be given in detail in Sec. IV. In essence $U /\left(K a^{2}\right)$ represents the ratio of substrate potential to elastic energy, such that when $U /\left(K a^{2}\right)=\pi^{2} \varepsilon^{2} / 16$ the stripe state of the Moiré pattern has the same energy as the commensurate phase in the small deformation limit. Expressing the results in terms of this dimensionless parameter removes the influence of the simulation parameters at small strains. Different choices of simulation parameter may quantitatively alter the large strain results but are unlikely to qualitatively change the basic phase diagrams shown later in this section.

The numerically determined phase diagram for the H-T systems is shown in Fig. 2(a). At small strains and adhesion
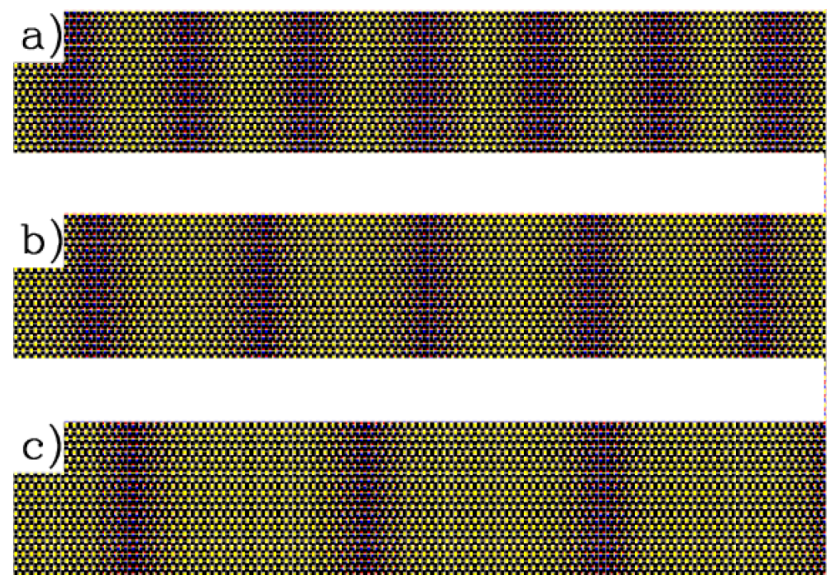

FIG. 4. Examples of metastable stripe patterns for the H-T system at $\varepsilon=5 \%$ and $U / K a^{2}=7.1876 \times 10^{-4}, 1.3872 \times 10^{-3}$, and $1.42890 \times 10^{-3}$ in (a)-(c), respectively.

strengths the lowest energy state is a triangular network of commensurate regions (or equivalently a honeycomb network of incommensurate domain walls), as illustrated in Fig. 3. When $U /\left(K a^{2}\right)$ is increased a first order phase transition to a completely commensurate state occurs. At larger strains a transition from a triangular to stripe phase occurs first, followed by a transition to the commensurate state for larger $U / K a^{2}$. As indicated by the dot in Fig. 2(a) a triple point exists

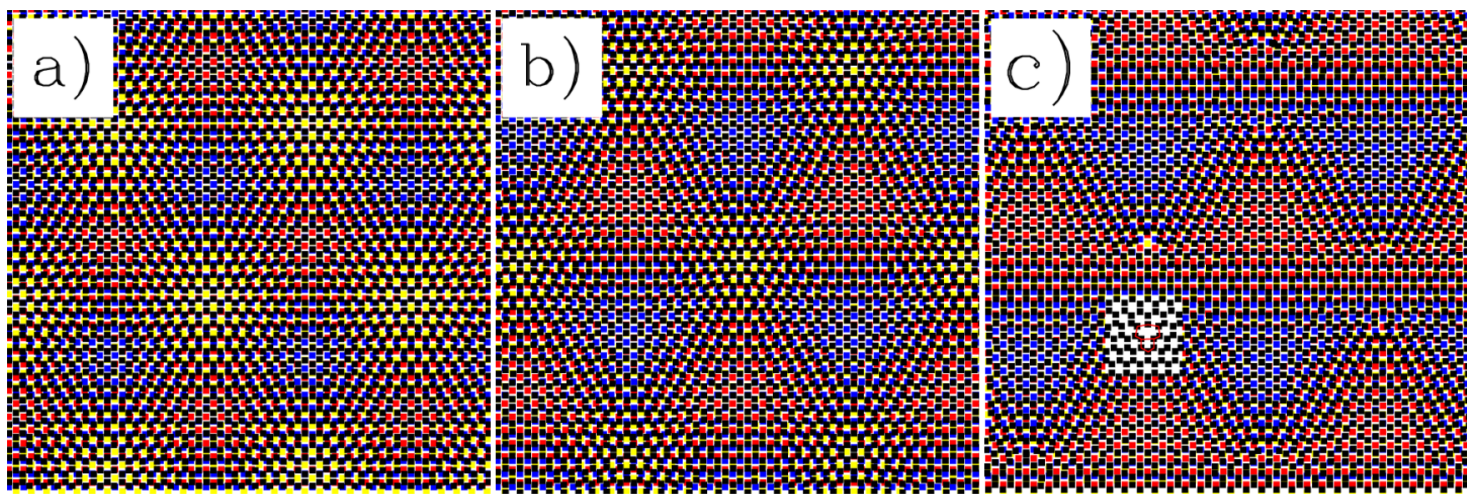

FIG. 5. Examples of equilibrium honeycomb Moiré patterns for the $\mathrm{H}-\mathrm{H}$ system at $\varepsilon=7 \%$ and $U / K a^{2}=7.9862 \times 10^{-4}$ and $3.5938 \times 10^{-3}$ in (a) and (b), respectively. In (c) a zigzag pattern is shown at $U / K a^{2}=4.9914 \times 10^{-3}$ and a defect is highlighted in the inset. In this figure the light (yellow) and dark (red and blue) areas correspond to incommensurate and commensurate regions, respectively. 

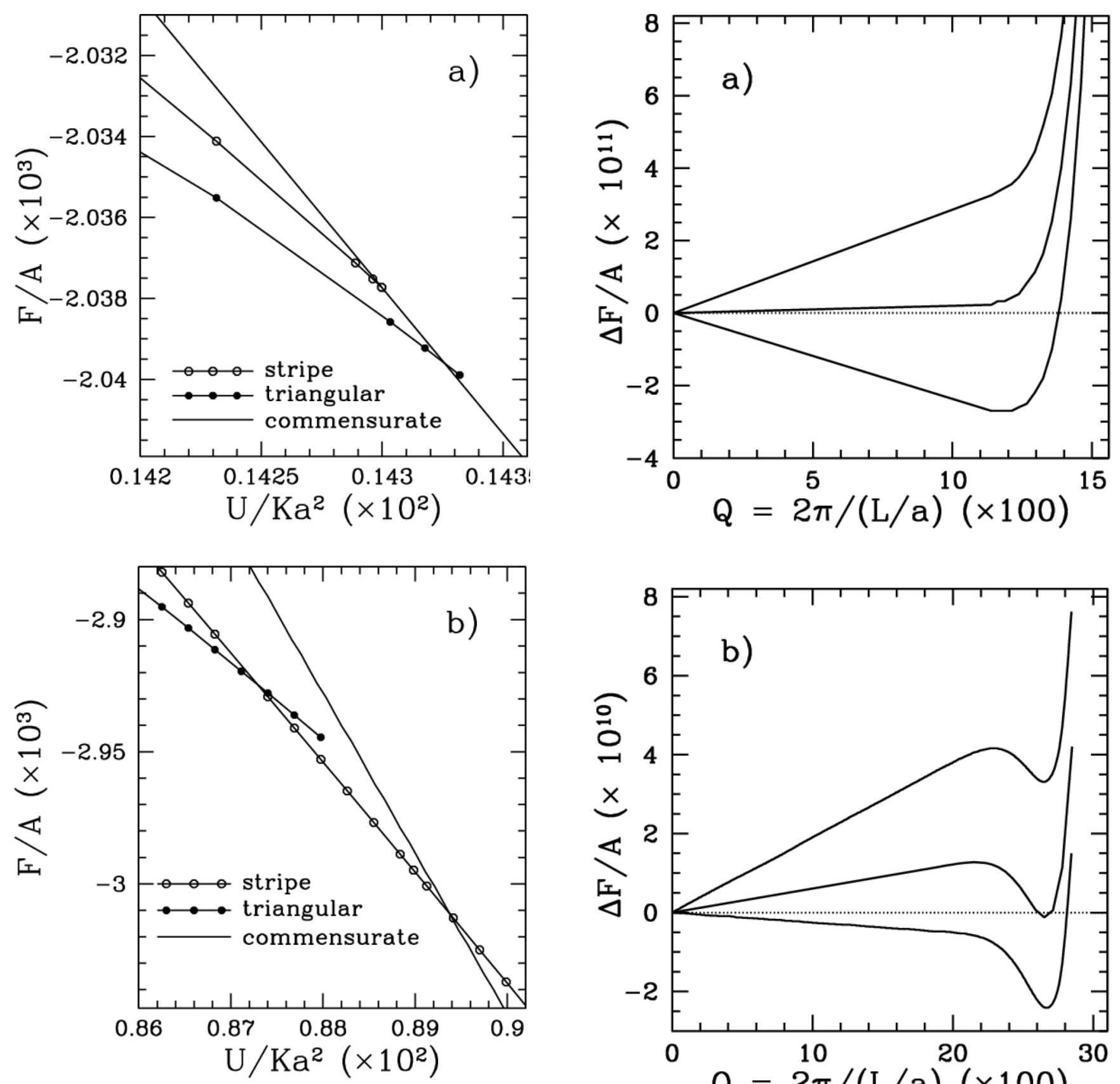

FIG. 6. Free energy per unit area as a function of $U / K a^{2}$ for the H-T system at strains of $5 \%$ and $13.5 \%$ in (a) and (b), respectively. The solid line, the solid line with open circles, and the solid line with filled circles correspond to commensurate, striped, and triangular states, respectively.

where the energy of the commensurate, striped, and triangular states is equal. Samples of the striped state are shown in Fig. 4. It is interesting to note that the stripe-commensurate transition is continuous for small strains (when the stripe state is metastable) and discontinuous or first order at larger strains as will be discussed later.

This behavior is considerably different for a $\mathrm{H}-\mathrm{H}$ (or T-T) system. For comparison the phase diagram for this system is shown in Fig. 2(b) (redrawn from Refs. 11 and 12). At very small adhesion strengths the commensurate regions form a honeycomb network as shown in Figs. 5(a) and 5(b). In between this honeycomb state and a completely commensurate state that appears at large enough adhesion, a transition to the

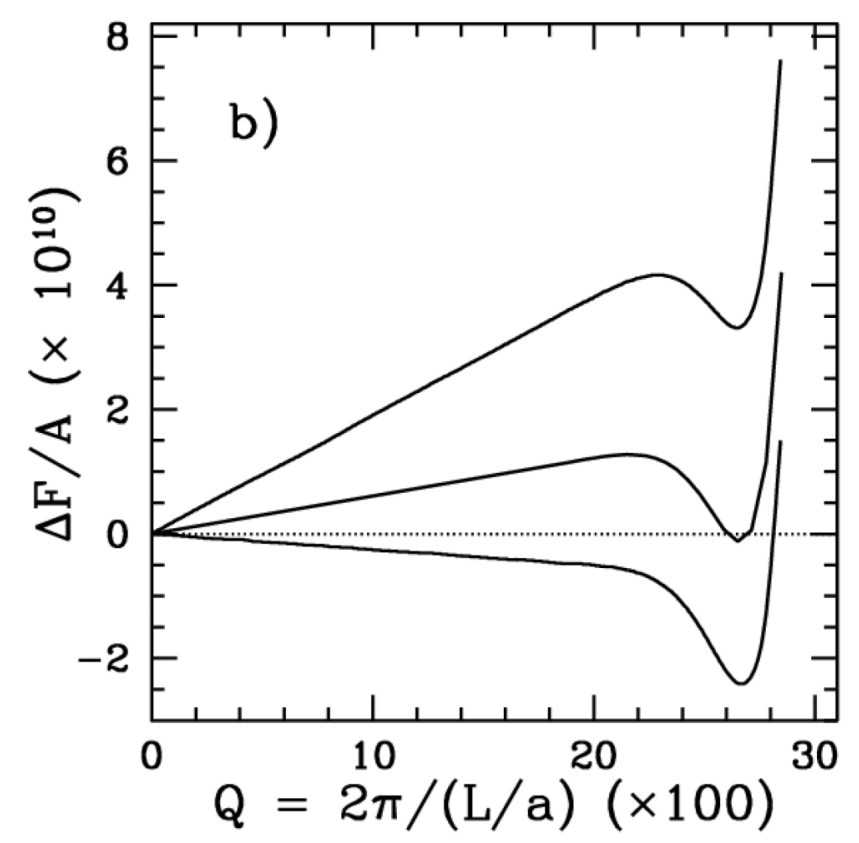

FIG. 7. Free energy difference, $\Delta F \equiv F(Q)-F(0)$, per unit area as a function of stripe wavenumber $Q$ for strains of $5 \%$ and $7 \%$ in (a) and (b), respectively. In (a) the lines from bottom to top correspond to $U / \mathrm{Ka}^{2}$ $=1.429985181 \times 10^{-3}, 1.429985468 \times 10^{-3}$, and $1.429985756 \times 10^{-3}$. In (b) the lines from bottom to top correspond to $U / K a^{2}=2.70371152 \times 10^{-3}$, $U / K a^{2}=2.70371295 \times 10^{-3}$, and $U / K a^{2}=2.70371511 \times 10^{-3}$.

stripe phase always occurs in contrast to the H-T case. At large strains and intermediate adhesion strengths a zigzag state (formerly referred to as a triangular state in Refs. 11 and 12) appears, consisting of an array of dislocation pairs as illustrated in Fig. 5(c). A triple point also occurs in this system where the zigzag, stripe, and honeycomb states can coexist. The dramatic difference between the H-T and $\mathrm{H}-\mathrm{H}$ systems can be attributed to the difference in energy at the domain wall junctions that form in the triangular (H-T) and honeycomb $(\mathrm{H}-\mathrm{H})$ patterns, as will be discussed in the next section. 
a)

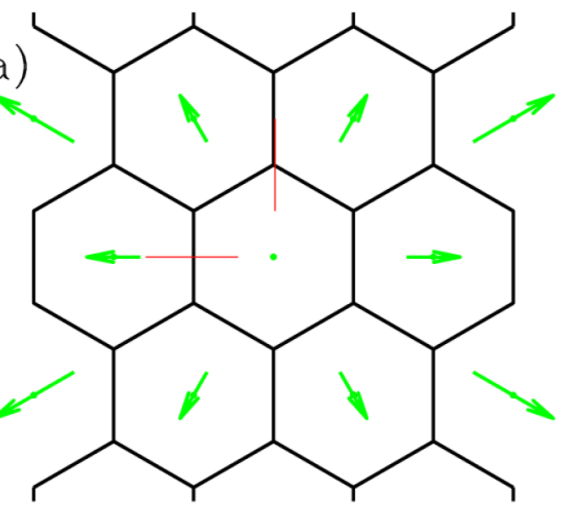

b)

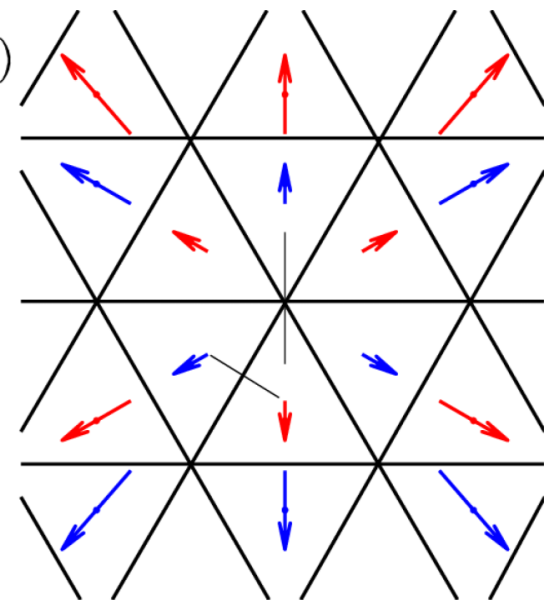

FIG. 8. Displacement vectors and domain walls for (a) H-T and (b) H-H systems. The arrows represent the direction and magnitude of the displacement vectors within each commensurate region The thin lines in the figures are guides to the eye for path across domain walls (lower lines) and through junctions (upper vertical lines).
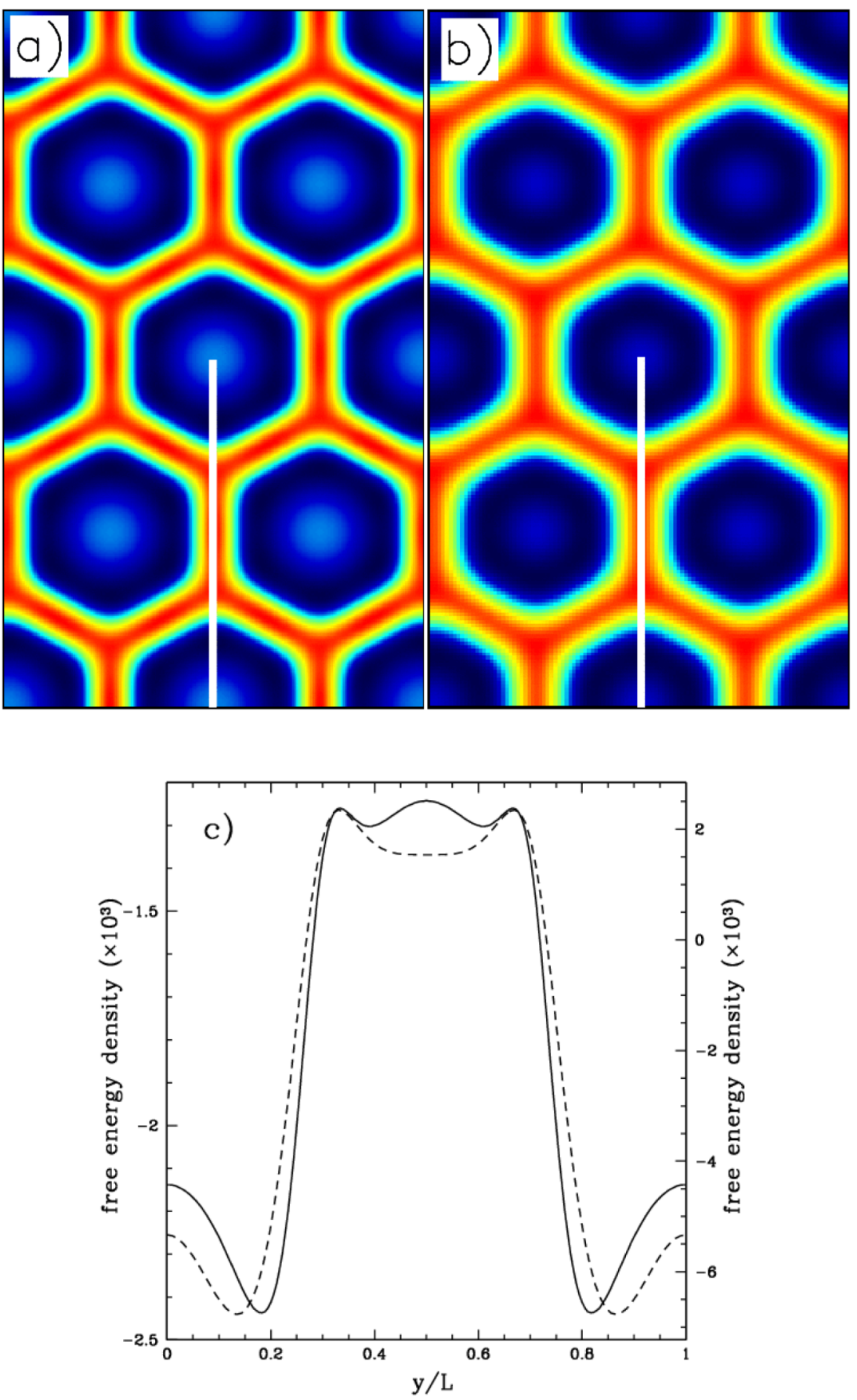

FIG. 9. Energy density profiles for H-T case at $\varepsilon=5 \%$ and $U / K a^{2}=1.3656$ $\times 10^{-3}$ for (a) and $\varepsilon=15 \%$ and $U / K a^{2}$ $=1.0350 \times 10^{-2}$ for (b). In (c) the energy density along the white lines in (a) and (b) is shown as the solid and dashed curves, respectively. The left (right) axis labels correspond to the solid (dashed) curve. In (a) and (b) red corresponds to higher energy density regions. 
All calculations were done in the absence of fluctuations, i.e., in the mean field limit. In this limit the numerical results indicate that in the H-T case the triangular to commensurate and the triangular to stripe transitions are both first order. The stripe to commensurate transition is second order for small strains and first order for large strains. Evidences of the first order transitions are given in Figs. 6 and 7. As seen in Fig. 6(a) the stripe phase at $\varepsilon=5 \%$ is never the lowest energy state and the free energy of this state never goes above the energy of the commensurate state. As further illustrated in Fig. 7, at a strain of $5 \%$ there appears to be a single minima at a finite wavelength, $L$, (or wavenumber $Q$ ), while at $\varepsilon=7 \%$ there are clearly two minima, with one at $Q=0$ (i.e., the commensurate state) and the other at a finite value of $Q$. In the latter case there exists a value of $U / K a^{2}$ for which the two minima have the same free energy per unit area. While the purpose of this work is not to accurately determine the precise strain at which the change in the nature of the stripecommensurate transition occurs the results indicate that the transition changes from continuous to discontinuous around a strain of $6 \%$. In the case of the $\mathrm{H}-\mathrm{H}$ (or T-T) system first order or discontinuous transitions were observed for the honeycomb-stripe, honeycomb-zigzag and zigzag-stripe transitions. Similar to the H-T case the stripe-commensurate transition was found to be continuous for strains up to $6 \%$ and discontinuous for larger strains.

\section{SMALL DEFORMATION LIMIT}

To understand the nature of surface ordering in these two systems it is useful to consider the limit of small deformations. In this limit the amplitudes can be represented as

$$
\eta_{k l m}=\phi e^{i \vec{G}_{k l m}^{f} \cdot \vec{u}}
$$

where $\vec{u}$ is the lattice displacement field used in standard continuum elasticity theory. For small $\vec{\nabla} \vec{u}$ the elastic and external potential contributions to the free energy given in Eq. (6) become

$$
\begin{aligned}
F_{u} \approx & \int d \vec{r}\left\{\frac{C_{11}}{2}\left[\left(u_{x x}-\varepsilon\right)^{2}+\left(u_{y y}-\varepsilon\right)^{2}\right]\right. \\
& +2 C_{44} u_{x y}^{2}+C_{12}\left(u_{x x}-\varepsilon\right)\left(u_{y y}-\varepsilon\right) \\
& \left.+2 V_{0} \phi \sum_{k l m} \cos \left(\vec{G}_{k l m}^{f} \cdot \vec{u}\right)\right\},
\end{aligned}
$$

where $u_{i j}=\left(\partial_{i} u_{j}+\partial_{j} u_{i}\right) / 2$ and the elastic constants are $C_{11}$ $=9 B^{x} \phi^{2}$ and $C_{12}=C_{44}=C_{11} / 3$. This form is similar to that used in previous studies of commensurate-incommensurate (C-I) transitions..$^{2-4}$ While this equation is difficult to solve in two dimensions for the boundary conditions of interest, it is possible to gain some insights into the nature of the two-dimensional patterns and to solve the one-dimensional stripe patterns exactly.

\section{A. Two-dimensional patterns}

In two dimensions the potential term in Eq. (11) has extrema at $u_{x}=n a / 2$ and $u_{y}=\sqrt{3} m a / 2$, where $n$ and $m$ are integers such that $n+m=$ even, and $a$ is the lattice constant shown in Fig. $1(a=4 \pi / \sqrt{3}$ in this system). In this instance the potential contribution to the free energy reduces simply to $6 V_{0} \phi$. In addition, the extrema also occur when $u_{x}=n a / 2$ and $u_{y}=a(\sqrt{3} m \pm 1 / \sqrt{3}) / 2$, where $n$ and $m$ are integers such that $n+m$ is odd, and the corresponding potential contribution is $-3 \phi V_{0}$. The \pm sign here corresponds to the two separate but equivalent sublattices. For the H-T system $\phi<0$ and $V_{0}>0$ so that $\left(\phi V_{0}\right)_{\mathrm{H}-\mathrm{T}}<0$, in contrast to the $\mathrm{H}-\mathrm{H}$ system in which $\phi<0$ and $V_{0}<0$ so that $\left(\phi V_{0}\right)_{\mathrm{H}-\mathrm{H}}>0$. These considerations

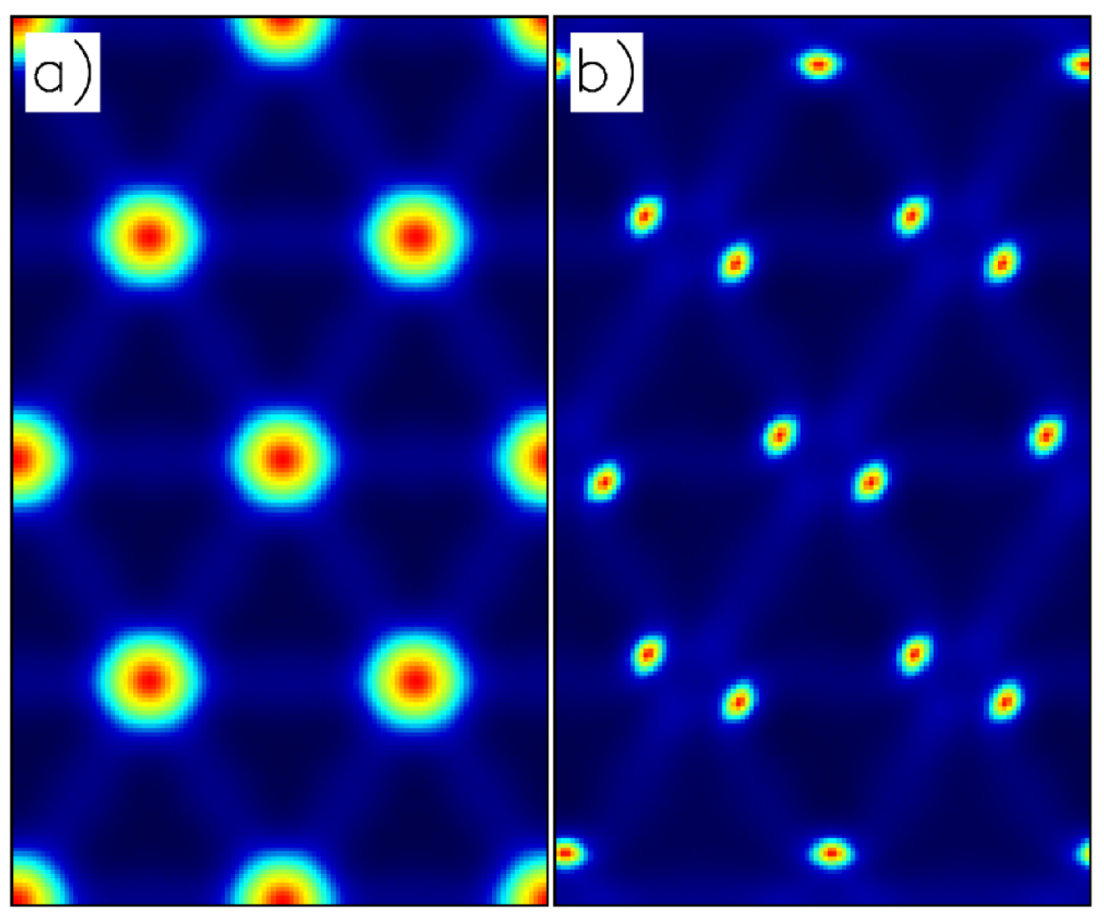

FIG. 10. Energy density profiles for $\mathrm{H}-\mathrm{H}$ case at $\varepsilon=7 \%$ and $U / K a^{2}$ $=3.9931 \times 10^{-4}$ for (a) and $U / K a^{2}$ $=4.9914 \times 10^{-4}$ for $(\mathrm{b})$. 

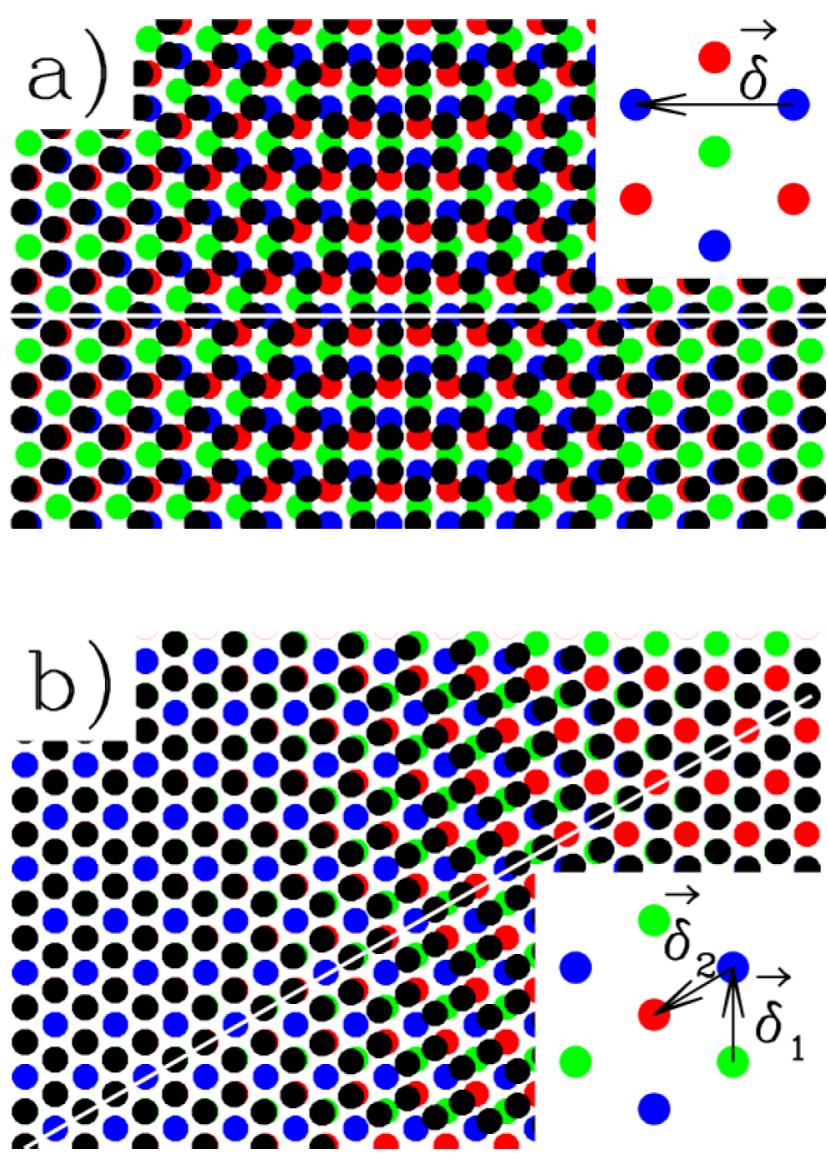

FIG. 11. Displacement across a domain wall in (a) H-T and (b) H-H systems.

imply the potential minima occur when

$$
\begin{array}{ccc}
\mathrm{H}-\mathrm{T}: & \left(u_{x}, u_{y}\right)=a(n, \sqrt{3} m) / 2, & n+m=\text { even, } \\
\mathrm{H}-\mathrm{H}: & \left(u_{x}, u_{y}\right)=a(n, \sqrt{3} m \pm 1 / \sqrt{3}) / 2, & n+m=\text { odd }
\end{array}
$$

The Moiré patterns that form in these systems are different due to the different numbers of commensurate states. In the H-T case the commensurate regions form a triangular pattern separated by a honeycomb network of domain walls as illustrated in Fig. 8(a). In contrast, in the H-H (or T-T) system the commensurate regions form a honeycomb pattern separated by a triangular network of domain walls as illustrated in Fig. 8(b). An interesting aspect of this comparison is the change in the displacement vector across a domain as opposed to that through a junction. In the $\mathrm{H}-\mathrm{T}$ case $|\Delta \vec{u}|$ across a domain wall is equal to $a$, while it is $\sqrt{3} a / 2$ through a junction (see the path shown in Fig. 8(a)). Thus the related contribution to the elastic energy is smaller when going through a junction, as compared to that across a domain wall. In the $\mathrm{H}-\mathrm{H}$ case the change is $a / \sqrt{3}$ across a domain wall and $2 a / \sqrt{3}$ through a junction, implying a much larger energy cost to the junction than the domain wall.

A comparison of domain wall energy can be obtained by examining the local free energy density (i.e., the integrand of Eq. (6)). This quantity is shown at two different strains for the H-T case in Fig. 9 and for the H-H case in Fig. 10(a). A comparison of these figures shows that the elastic energy at junctions is much larger in the H-H system compared to that in the H-T case, which explains why the striped phase
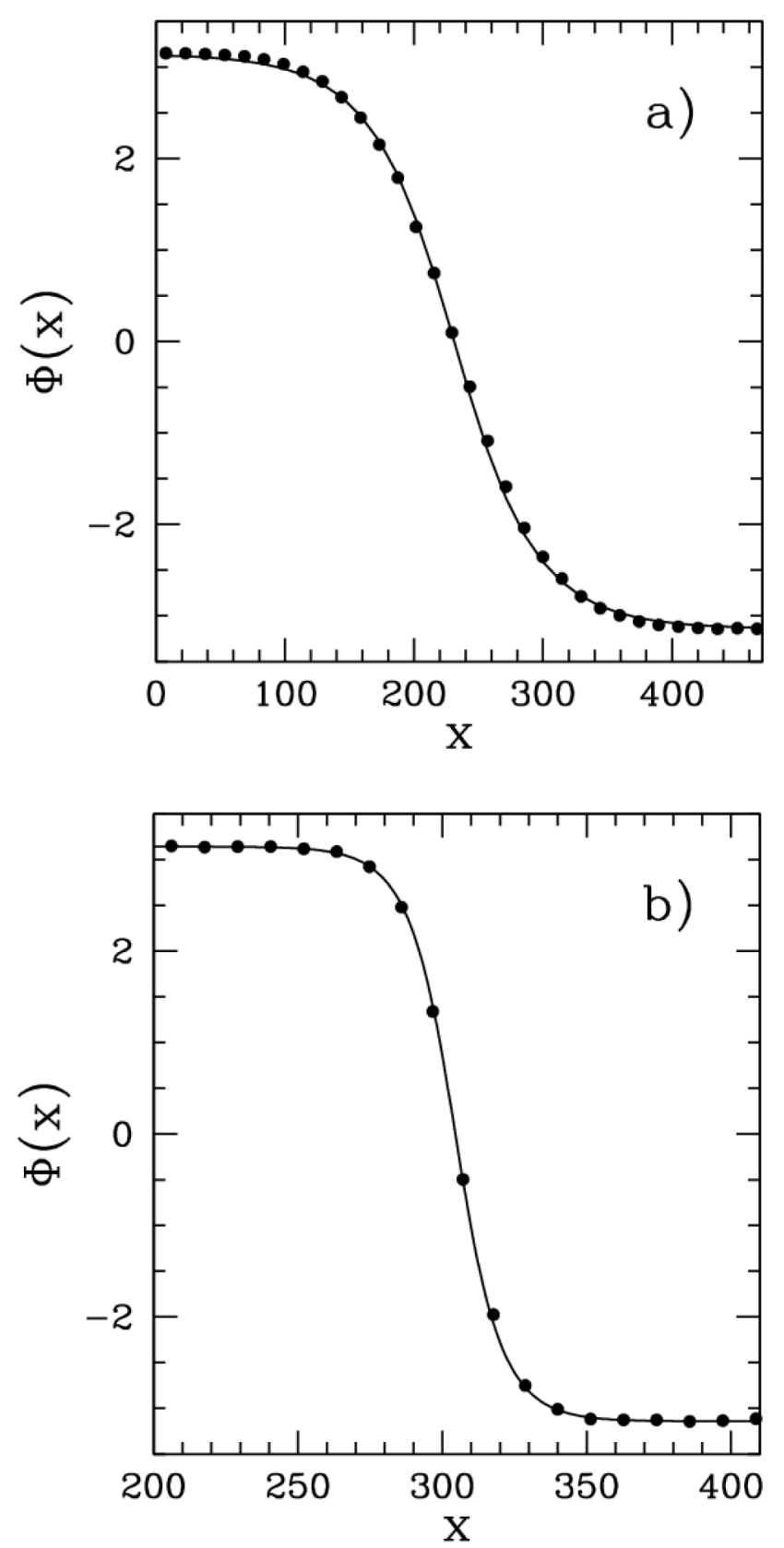

FIG. 12. A kink profile of domain wall (points) is compared with the analytic form given in Eq. (16) (line), at $\varepsilon=5 \%$ and $U / K a^{2}=1.429 \times 10^{-3}(\mathrm{H}-\mathrm{T})$ and $1.732 \times 10^{-3}(\mathrm{H}-\mathrm{H})$ for (a) and (b), respectively. The best fit values of $\lambda$ are 20.4 and 10.2 for (a) and (b), respectively, compared with theoretical values of 22.8 and 8.5 .

could occur in the phase diagram for all strains in the $\mathrm{H}-\mathrm{H}$ system (with small enough adhesion strength). In Fig. 9(c) the free energy density is plotted along a domain wall and through a junction for two different strains. As can be seen in this figure for small strains there is a slight dip in the energy at the junction, indicating that the junctions cost less energy than domain walls. This is consistent with the absence of the striped state in the H-T case at low strains. This figure also shows that at higher strains (dashed curve) the junction energy is higher than the domain wall energy, which explains why the striped phase appears in the phase diagram at high misfit strains. Figure 10(b) shows the free energy density in 

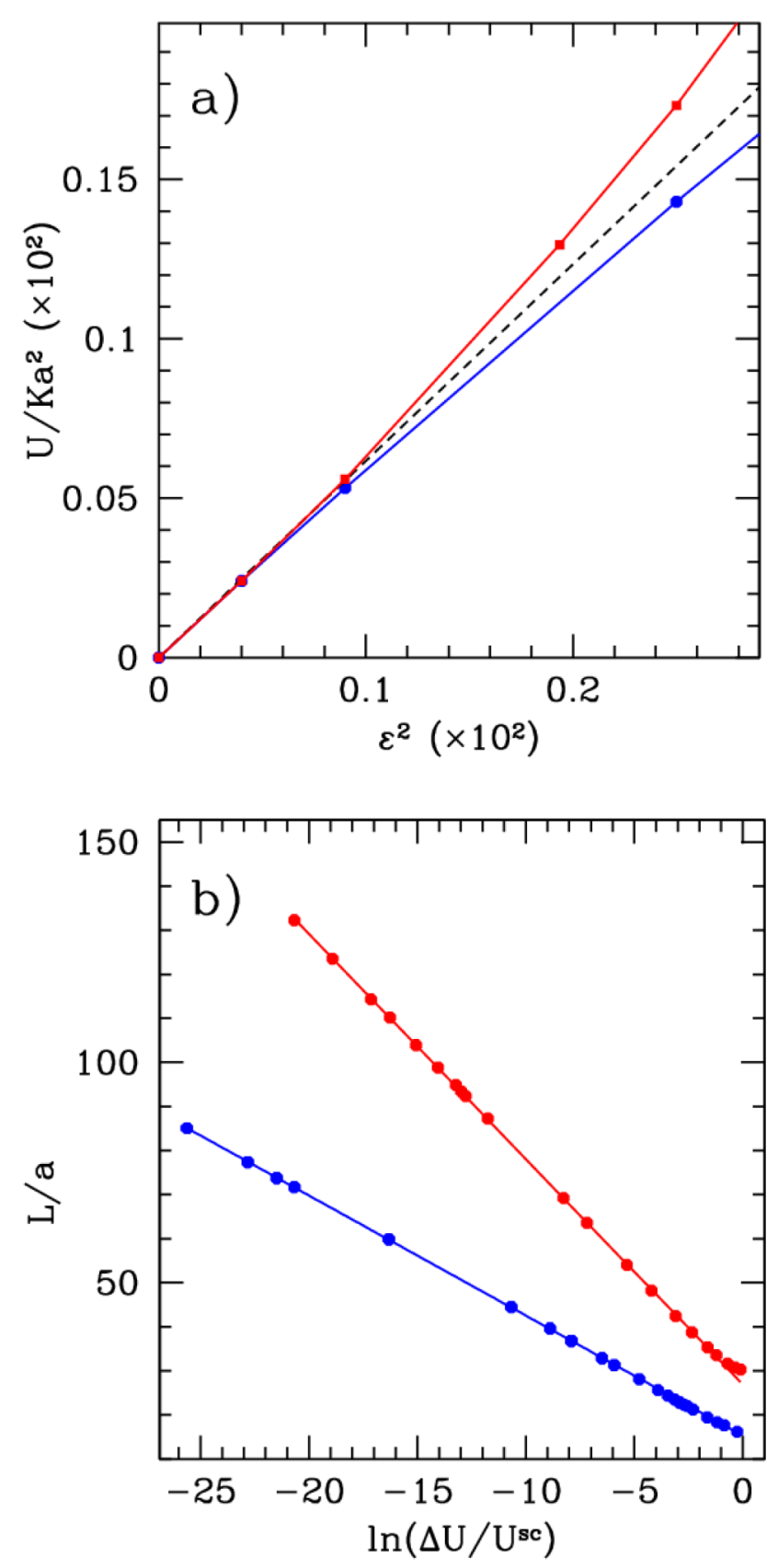

FIG. 13. In (a) comparison of numerical and analytic (dashed black line) results for $\mathrm{H}-\mathrm{H}$ (red square points) and H-T (blue circular points) stripecommensurate transitions. In (b) the stripe wavelength is shown as a function of $\ln \left(\Delta U / U^{s c}\right)$. The slopes for H-H and H-T cases are $\lambda=18.6$ and 9.9, respectively, compared to analytic predictions of 22.8 and 8.7.

the zigzag region of the $\mathrm{H}-\mathrm{H}$ phase diagram, which contains a periodic array of dislocations. Essentially the junction energy becomes so large that it is preferable for the junctions to split into dislocation pairs.

\section{B. One-dimensional stripes}

While it is difficult to solve Eq. (11) for the twodimensional Moiré patterns, it can be solved exactly for the one-dimensional stripe phase. The stripe superstructures can be described by a one-dimensional displacement vector of the form $\vec{u}=(\Phi(x) / 2 \pi) \vec{\delta}$ in the H-T system, where $\vec{\delta}=-a \hat{x}$, and for the H-H case $\vec{u}=\vec{\delta}_{1}+(\Phi(x) / 2 \pi) \vec{\delta}_{2}$ with $\vec{\delta}_{1}=a / \sqrt{3} \hat{y}$ and $\vec{\delta}_{2}=-a(\hat{x}+\hat{y} / \sqrt{3}) / 2$, as shown in Fig. 11. Eq. (11) can then be transformed to the well-known sine-Gordon free energy

$$
F_{u}^{1 d} \approx \int d \vec{r}\left[\frac{K}{2}\left(\frac{\partial \Phi}{\partial x}-\varepsilon\right)^{2}+\frac{U}{a^{2}} \cos \Phi\right],
$$

where the elastic modulus $K$ is given by

$$
K=\left\{\begin{array}{cc}
\left(C_{11}+C_{12}\right)^{2} / C_{11} & \mathrm{H}-\mathrm{T} \\
\left(C_{11}+C_{12}\right)^{2} /\left(C_{11}+C_{44} / 3\right) & \mathrm{H}-\mathrm{H}
\end{array},\right.
$$

and the adhesion energy $U$ is

$$
\frac{U}{a^{2}}=\left\{\begin{array}{cc}
4 V_{0} \phi & \mathrm{H}-\mathrm{T} \\
V_{0} \phi / 2 & \mathrm{H}-\mathrm{H}
\end{array} .\right.
$$

Equation (12) can be solved exactly ${ }^{4,5,11}$ near the stripecommensurate transition which occurs when the ratio of adhesion to elastic energy is equal to $\pi^{2} / 16$, i.e.,

$$
U^{s c}=\frac{\pi^{2}}{16} K a^{2} \varepsilon^{2} .
$$

The kink profile for the domain walls is given by

$$
\Phi(x)=2 \sin ^{-1}[\tanh (x / \lambda)],
$$

where $\lambda=\sqrt{K a^{2} / U}$ is the domain wall width. When approaching the transition point the stripe wavelength diverges as $L=-2 \lambda \ln \left(\Delta U / U^{s c}\right)+$ constant, with $\Delta U=U^{s c}-U$. A comparison of the prediction of Eq. (16) and domain wall profiles obtained numerically is given in Fig. 12, showing a very good agreement for both $\mathrm{H}-\mathrm{T}$ and $\mathrm{H}-\mathrm{H}$ cases.

At small $\varepsilon$ these analytic predictions agree very well with the numerical results, as shown in Fig. 13 for critical stripecommensurate transition. At larger strains the qualitative predictions of a continuous phase transition with a diverging $L$ for both $\mathrm{H}-\mathrm{T}$ and $\mathrm{H}-\mathrm{H}$ systems are still accurate up to roughly $\varepsilon \approx 6 \%$, as seen, for example, in Fig. 13(b) for $L$ and Fig. 12 for $\Phi(x)$. For large enough $\varepsilon$ the prediction of a continuous stripe-commensurate transition breaks down, as evidenced in Figs. 6(b) and 7(b).

\section{DISCUSSION AND CONCLUSIONS}

The main difference between the phase diagrams of the $\mathrm{H}-\mathrm{T}$ and $\mathrm{H}-\mathrm{H}$ systems can be attributed to the nature of the junctions or domain wall crossing in the two systems. In the former case the energy at a junction is similar to that of a domain wall, whereas in the latter case the junction energy is significantly higher than at a domain wall. This occurs as the change in the displacement vector through a junction is much larger in the H-H system than in the H-T case. At small strains this difference results in the appearance of the striped state at relatively low adhesion strengths in the $\mathrm{H}-\mathrm{H}$ system and a direct transition from a triangular Moiré pattern to the commensurate state in the H-T system. The transition in the latter case is discontinuous. In principle it is possible for the triangular-commensurate transition to be continuous since a triangular pattern can continuously change into the commensurate state by increasing its length scale to infinity. 
However, this continuous transition can never occur because at some point the striped state would have a lower energy, given that it has a lower density of domain walls than the triangular state and the importance of the junctions diminishes as the length scale of the patterns increases.

For the other discontinuous transitions most are straightforward to understand. For example, the triangularstripe, honeycomb-stripe, honeycomb-zigzag, and zigzagstripe transitions are discontinuous since there is a distinct change of pattern symmetry and hence it is impossible to evolve continuously from one state to another. However, this does not apply to the stripe-commensurate transition as a commensurate state is simply a stripe state with infinite wavelength. While there is no a priori reason to believe that this implies the transition must be continuous, this is what is observed at low strains and in the prediction of the simplified sine-Gordon model. At large strains the stripe-commensurate transition is found to be discontinuous in both H-T and H-H systems. This is likely due to the discrete nature of the crystalline lattice, which is captured by the amplitude description used in this work but lost in the continuum approximation leading to Eq. (11) and in previous studies using continuum elasticity theory and the sine-Gordon formulation. This breakdown at large $\varepsilon$ is not unexpected since the nominal Moiré length scale at zero $V_{0}$ is given by $a_{f} / \varepsilon$ (where $a_{f}$ is the film lattice constant), which becomes small for large $\varepsilon$.

While the predictions of this work rely on a relatively simple model (i.e., a 2D complex amplitude description coupled to a fixed substrate potential), the results are mainly based on basic symmetry arguments and the competition between strain and adhesion energy. As such the fundamental features of the phase diagrams identified should be applicable to other models and systems. It is also important to note that the method adopted here incorporates both microscopic and mesoscopic descriptions and thus is able to simultaneously capture the large-scale features of the complex superstructures that emerge as well as resolve the atomistic and discrete nature of the underlying crystalline lattices. To compare the results of this work with a given physical system the parameter $U / K a^{2}$ needs to be evaluated. The parameter $K$ can be evaluated by knowledge of the elastic constant through Eq. (13). The parameter $U$ is related to the magnitude of the surface potential magnitude $V_{0}$ through Eq. (14). This parameter can be related to the interaction energy of the film with respect to the substrate, which can be calculated from density functional theory. ${ }^{11}$ The validity of this modeling scheme has been verified in the previous work for the T-T system where quantitative agreements with experiments of $\mathrm{Cu} / \mathrm{Ru}(0001)$ and $\mathrm{Cu} / \mathrm{Pd}(111)$ were obtained. ${ }^{11}$ For some H-T systems such as graphene or h-BN layers on metallic surfaces, typically the coupling between film and substrate is quite weak and thus the results of this work are relevant to these graphenetype materials at very small $V_{0}$, leading to the emergence of triangular Moiré patterns as observed experimentally. ${ }^{17,18}$ To further validate the $\mathrm{H}-\mathrm{T}$ results particularly those at large $V_{0}$, sample simulations of a three-mode PFC model for honeycomb lattice ${ }^{36}$ were also conducted. The outcomes were consistent with the predictions described above in particular the phenomena of transitions among triangular, stripe, and commensurate phases. It should be noted that very close to the continuous stripe-commensurate transitions fluctuations (not considered in this work) may alter the nature of the transition. It would be interesting to experimentally test the predictions of this work and to extend the modeling approach to a broader range of systems that are of experimental relevance.

\section{ACKNOWLEDGMENTS}

This work was in small part supported by the Academy of Finland through its Centres of Excellence Programme (Grant Nos. 2012-2014 and 2015-2017) under Project Nos. 251748 and 284621, the National Science Foundation under Grant No. DMR-1506634 (K.R.E.), the National Science Foundation under Grant No. DMR-0845264 (Z.-F.H.), the Watson Institute at Brown University under a Brazil Collaborative Grant (S.-C.Y), the National Natural Science Foundation of China Grant No. 11102164 (Z.C.), and the São Paulo Research Foundation (FAPESP, Grant No. 2014/15372-3) (E.G.).

${ }^{1}$ P. Bak, Rep. Prog. Phys. 45, 587 (1982).

${ }^{2}$ J. H. van der Merwe, J. Appl. Phys. 41, 4725 (1970).

${ }^{3}$ S. C. Ying, Phys. Rev. B 3, 4160 (1971).

${ }^{4}$ P. Bak, D. Mukamel, J. Villain, and K. Wentowska, Phys. Rev. B 19, 1610 (1979).

${ }^{5}$ P. M. Chaikin and T. C. Lubensky, Principles of Condensed Matter Physics (Cambridge University Press, Cambridge, 1995).

${ }^{6}$ F. E. Gabaly, W. L. W. Ling, K. F. McCarty, and J. de la Figuera, Science 308, 1303 (2005).

${ }^{7}$ C. Günther, J. Vrijmoeth, R. Q. Hwang, and R. J. Behm, Phys. Rev. Lett. 74, 754 (1995).

${ }^{8}$ S.-F. Ding, S.-R. Deng, H.-S. Lu, Y.-L. Jiang, G.-P. Ru, D. W. Zhang, and X. P. Qu, J. Appl. Phys. 107, 103534 (2010).

${ }^{9}$ M. Wasniowska, W. Wulfhekel, M. Przybylski, and J. Kirschner, Phys. Rev. B 78, 035405 (2008).

${ }^{10}$ J. Jalkanen, G. Rossi, O. Trushin, E. Granato, T. Ala-Nissila, and S.-C. Ying, Phys. Rev. B 81, 041412 (2010).

${ }^{11}$ K. R. Elder, G. Rossi, P. Kanerva, F. Sanches, S.-C. Ying, E. Granato, C. V. Achim, and T. Ala-Nissila, Phys. Rev. Lett. 108, 226102 (2012).

${ }^{12}$ K. R. Elder, G. Rossi, P. Kanerva, F. Sanches, S.-C. Ying, E. Granato, C. V. Achim, and T. Ala-Nissila, Phys. Rev. B 88, 075423 (2013).

${ }^{13}$ K. L. Man, M. C. Tringides, M. M. T. Loy, and M. S. Altman, Phys. Rev. Lett. 110, 036104 (2013).

${ }^{14}$ E. Granato, S. C. Ying, K. R. Elder, and T. Ala-Nissila, Phys. Rev. Lett. 111, $126102(2013)$

${ }^{15}$ O. V. Yazyev and Y. P. Chen, Nat. Nanotechnol. 9, 755 (2014).

${ }^{16}$ S. Z. Butler et al., ACS Nano 7, 2898 (2013).

${ }^{17}$ N. C. Bartelt and K. F. McCarty, MRS Bull. 37, 1158 (2012).

${ }^{18}$ S. Marchini, S. Günther, and J. Wintterlin, Phys. Rev. B 76, 075429 (2007).

${ }^{19}$ S. Nie, J. M. Wofford, N. C. Bartelt, O. D. Dubon, and K. F. McCarty, Phys. Rev. B 84, 155425 (2011).

${ }^{20}$ R. Drost, A. Uppstu, F. Schulz, S. K. Hamalainen, M. Ervasti, A. Harju, and P. Liljeroth, Nano Lett. 14, 5128 (2014).

${ }^{21}$ N. Goldenfeld, B. P. Athreya, and J. A. Dantzig, Phys. Rev. E 72, 020601 (2005).

${ }^{22}$ B. P. Athreya, N. Goldenfeld, and J. A. Dantzig, Phys. Rev. E 74, 011601 (2006).

${ }^{23}$ B. P. Athreya, N. Goldenfeld, J. A. Dantzig, M. Greenwood, and N. Provatas, Phys. Rev. E 76, 056706 (2007).

${ }^{24}$ D.-H. Yeon, Z.-F. Huang, K. R. Elder, and K. Thornton, Philos. Mag. 90, 237 (2010).

${ }^{25}$ K. R. Elder, Z.-F. Huang, and N. Provatas, Phys. Rev. E 81, 011602 (2010).

${ }^{26}$ Z.-F. Huang, K. R. Elder, and N. Provatas, Phys. Rev. E 82, 021605 (2010).

${ }^{27}$ K. R. Elder, M. Katakowski, M. Haataja, and M. Grant, Phys. Rev. Lett. 88, 245701 (2002).

${ }^{28}$ K. R. Elder and M. Grant, Phys. Rev. E 70, 051605 (2004). 
${ }^{29}$ K. R. Elder, N. Provatas, J. Berry, P. Stefanovic, and M. Grant, Phys. Rev. B 75, 064107 (2007).

${ }^{30}$ N. Provatas and K. R. Elder, Phase-Field Methods in Materials Science and Engineering (Wiley-VCH, Weinheim, 2010).

${ }^{31}$ Z.-F. Huang and K. R. Elder, Phys. Rev. Lett. 101, 158701 (2008).

${ }^{32}$ Z.-F. Huang and K. R. Elder, Phys. Rev. B 81, 165421 (2010).

${ }^{33}$ J. Mellenthin, A. Karma, and M. Plapp, Phys. Rev. B 78, 184110 (2008)

${ }^{34}$ A. Jaatinen, C. V. Achim, K. R. Elder, and T. Ala-Nissila, Phys. Rev. E 80, 031602 (2009).
${ }^{35}$ E. J. Schwalbach, J. A. Warren, K.-A. Wu, and P. W. Voorhees, Phys. Rev. E 88, 023306 (2013).

${ }^{36}$ S. K. Mkhonta, K. R. Elder, and Z.-F. Huang, Phys. Rev. Lett. 111, 035501 (2013).

${ }^{37}$ C. V. Achim, M. Schmiedeberg, and H. Löwen, Phys. Rev. Lett. 112, 255501 (2014).

${ }^{38}$ K.-A. Wu, C.-H. Wang, J. J. Hoyt, and A. Karma, Phys. Rev. B 91, 014107 (2015).

${ }^{39}$ G. Kocher and N. Provatas, Phys. Rev. Lett. 114, 155501 (2015). 